Meta

Journal des tradlucteurs

Translators' Journal

\title{
Samuel Beckett : traducteur de lui-même
}

\section{Blake T. Hanna}

Volume 17, numéro 4, décembre 1972

URI : https://id.erudit.org/iderudit/003005ar

DOI : https://doi.org/10.7202/003005ar

Aller au sommaire du numéro

Éditeur(s)

Les Presses de l'Université de Montréal

ISSN

0026-0452 (imprimé)

1492-1421 (numérique)

Découvrir la revue

Citer cet article

Hanna, B. T. (1972). Samuel Beckett : traducteur de lui-même. Meta, 17(4),

220-224. https://doi.org/10.7202/003005ar d'utilisation que vous pouvez consulter en ligne.

https://apropos.erudit.org/fr/usagers/politique-dutilisation/ 


\section{Samuel Beckett}

\section{traducteur de lui-même *}

Les auteurs dont la connaissance de deux langues leur permet de laisser une empreinte sur deux littératures sont rares et ceux d'entre eux qui traduisent leurs propres œuvres le sont davantage. Tel pourtant est le cas de Samuel Beckett, auteur de En attendant Godot, de Krapp's Last Tape et de nombreux autres ouvrages, composés tantôt en français et tantôt en anglais. La plupart de ses ouvrages ont été traduits dans l'autre langue par Beckett lui-même, travaillant seul ou en collaboration. Certains d'entre eux, dont la traduction des deux pièces mentionnées : Waiting for Godot et la Dernière Bande, ont connu en traduction un succès à l'égal de celui de la version originale. La thèse de $\mathrm{M}$. Simpson analyse la situation assez particulière de Beckett pour en tirer des conclusions intéressant la litttérature comparée, l'étude de la traduction et celle du bilinguisme.

L'œuvre littéraire ou dramatique, comme toute autre création artistique d'ailleurs, est l'extériorisation d'une pensée que l'auteur cherche à livrer à son auditoire. Il s'agit donc d'une réalité bidimensionnelle où chaque idée est livrée dans son enveloppe particulière ou bien, en termes saussuriens, où chaque signifié retrouve son signifiant. Or, dans ce monde littéraire bidimensionnel, nous fait remarquer M. Simpson, Beckett nous offre une œuvre à trois dimensions, puisqu'il dispose de deux enveloppes, plutôt que d'une seule. C'est la raison pour laquelle la plupart des critiques le lisent dans l'original et en traduction avant de porter un jugement. Il y a en effet trois Godot, continue le candidat : la pièce en français, la pièce en anglais et le prototype qui n'existe que dans l'esprit de l'auteur.

Mais où se situe le traducteur devant cette réalité double? En effet, tout son art se déploie dans le jeu des signifiants, car il substitue à l'enveloppe fournie par l'auteur une autre enveloppe de sa propre confection dans l'espoir que celle-ci convienne à son contenu aussi étroitement que celle-là. S'il réussit, son intervention

* Guy Ekundayo Simpson, le Bilinguisme de Samuel Beckett d'après ses traductions du français à l'anglais et vice versa, thèse présentée à l'École des gradués de l'Université Laval en vue de l'obtention du doctorat en études françaises, Québec, Université Laval, xxxii +291 p., index des notions et index des mots français et anglais. 
passe inaperçue ; s'il échoue, ses critiques se mettent à citer la maxime Traduttore, traditore. Mais l'intérêt de Beckett pour le traducteur est qu'il fait disparaître certaines variables difficilement contrôlables. Par exemple, l'auteur et le traducteur étant la même personne, on évite l'intervention d'un deuxième esprit avec ses risques d'interprétation intrusive. Et pour la même raison, la traduction n'est plus subordonnée à la version originale, puisque original et traduction doivent être considérés comme des manifestations également authentiques de la pensée de l'auteur.

Dans ces conditions, est-il réellement juste de parler de traduction? Sans doute que non, du moins dans le sens traditionnel du terme, car si Waiting for Godot, pour reprendre l'exemple de tout à l'heure, est la traduction de En attendant Godot, il serait aussi faux de considérer la version française plus authentique que la version anglaise que de soutenir le contraire, à savoir que, parce que sa rédaction est postérieure à celle de la version française, Waiting for Godot aurait été destiné à remplacer En attendant Godot. Il s'agit tout simplement de deux versions de la même pièce, issues du même cerveau, conçues pour deux auditoires différents et destinées par l'auteur à coexister.

Le grand mérite de $\mathrm{M}$. Simpson est d'avoir compris qu'en éliminant le traducteur et en groupant ses textes deux par deux en versions de prestige égal, l'œuvre de Samuel Beckett lui fournissait une occasion sans pareille de vérifier certaines théories sur l'anglais et le français fondées sur le travail des traducteurs classiques. Sa thèse en dit aussi long sur les deux langues en cause que sur Beckett lui-même. Certaines équivalences inventées par l'auteur, par exemple, sont de nature à conseiller une prudence extrême au traducteur qui chercherait à se tirer d'une mauvaise passe en invoquant l'excuse du «jeu de mots intraduisible». Considérons les passages suivants, évoqués par M. Simpson :

cLov. : À moins qu'elle ne se tienne coïte.

HAMM. : Coïte ! Coite tu veux dire. À moins qu'elle ne se tienne coite.

cLov. : Ah ! On dit coite ? On ne dit pas coïte?

cLov. : ... Unless he's laying doggo.

HAMM. : Laying! Lying you mean. Unless he's lying doggo.

cLov. : Ah ! One says lying ? One doesn't say laying ${ }^{1}$ ?

Et, sortie de la même inspiration, une anecdote tirée de la même pièce touchant un tailleur et son client qui se fait faire un pantalon :

Sorry, revenez dans huit jours, j’ai raté le fond.

Bon, ça va, le fond, c'est pas commode.

[....]

Désolé, revenez dans dix jours, j'ai salopé l'entre-jambes.

Bon, d'accord, l'entre-jambes, c'est délicat.

[....]

J'ai bousillé la braguette.

Bon, à la rigueur, une belle braguette, c'est calé.

Sorry, come back in a week, I've made a mess of the seat.

Good, a neat seat can be very ticklish.

[...]

Frightfully sorry, I've made a hash of the crotch.

1. Fin de partie, Paris, Editions de Minuit, 1957, p. 50-51; Endgame, New York, Grove Press, 1958, p. 33-34. 
Good, can't be helped, a snug crotch is always a teaser.

[...]

I made a balls of the fly.

Good, at a pinch, a smart fly is a stiff proposition 2 .

Comme on peut l'imaginer, le client finit par se fâcher.

Dans son ensemble, la thèse vérifie les théories avancées dans la Stylistique comparée du français et de l'anglais ${ }^{3}$, dont l'un des auteurs, M. Jean Darbelnet, a dirigé les recherches de $M$. Simpson. C'est ainsi que le candidat retrouve dans l'œuvre de Beckett des exemples d'emprunt :

Sorry, revenez dans huit jours ;

Sorry, come back in a week ;

de traduction littérale :

Tu n'as jamais eu la curiosité, pendant que je dormais, d'enlever mes lunettes et de regarder mes yeux?

Did you never have the curiosity, while I was sleeping, to take off my glasses and look at my eyes 4 ?

de transposition :

... back and forth, in and out of someone's eye ;

... allant et venant, passant et repassant, dans l'œil de quelqu'un 5 ;

de modulation :

Was I lovable once?

Fut-il jamais un temps où je pouvais séduire ${ }^{6}$ ?

(II s'agit d'une modulation de cause à effet. Le candidat cite également des exemples de modulations impliquant un changement de point de vue, un renversement de termes, un changement de symboles ou le contraire négativé, ainsi que des exemples prenant l'abstrait pour le concret ou vice versa, une partie pour le tout ou vice versa, une partie pour une autre, ou bien substituant la métaphore à la vue directe.)

d'équivalence :

... don't stand on ceremony ;

... pas de chichis 7 ;

et finalement, d'adaptation :

Normandie 8 .

Connemara 9 .

Ayant discuté des différents procédés de traduction, M. Simpson passe à l'étude de deux aspects de la stylistique devant lesquels le français et l'anglais réagissent différemment : l'utilisation des mots-images et des mots-signes d'une

2. Fin de partie, p. 37 ; Endgame, p. 22.

3. Jean-Paul Vinay et Jean Darbelnet, Stylistique comparée du français et de l'anglais : méthode de traduction; nouv. éd. rev. et corr., Montréal, Beauchemin, 1970.

4. Fin de partie, p. $18 ;$ Endgame, p. 3 .

5. Happy Days, New York, Grove Press, 1961, p. 40 ; Oh! les beaux jours, Paris, Editions de Minuit, 1963 , p. 54.

6. Happy Days, p. 31 ; Oh! les beaux jours, p. 42.

7. Happy Days, p. 63 ; Oh! les beaux jours, p. 87.

8. En attendant Godot, Paris, Editions de Minuit, 1952, p. 75.

9. Waiting for Godot, New York, Grove Press, 1954, p. 29. 
part, et d'autre part, la tendance dynamique opposée à la tendance statique. Sur ce point encore, ses constatations confirment celles de Vinay et Darbelnet.

Dans une première série d'exemples tirés de l'œuvre de Beckett, M. Simpson démontre que là où l'anglais s'attache au concret, le français s'intéresse à l'abstrait. Voici un exemple illustrant cette tendance :

... il se sauve au plus profond des taillis.

... he slinks away into the depths of the thicket 10 .

Dans une deuxième série d'exemples, $M$. Simpson trouve dans l'œuvre de Beckett de nombreux passages qui confirment la tendance de l'anglais à saisir l'aspect dynamique de l'action, tandis que le français adopte à son égard une attitude statique. Là où l'anglais suit fidèlement le déroulement de l'action, le français la saisit sous un jour complètement différent. Dans certains cas, cette dernière langue identifie d'abord l'action, pour l'analyser ensuite :

Il vide sa pipe en la tapant contre son fouet.

He knocks out his pipe against the whip 11 (chassé-croisé).

Dans d'autres cas, le français se contente de n'exprimer que l'essentiel de l'action, laissant à l'anglais le soin de la suivre jusque dans ses détails :

The parasol slips from her grasp.

L'ombrelle lui échappe 12 (économie par évidence).

Dans d'autres cas encore, le français analyse l'action, plutôt que d'en suivre le déroulement chronologique :

... these things tide one over.

... avec ça on peut voir venir ${ }^{13}$.

Et finalement, l'évidence offerte par l'œuvre de Beckett témoigne abondamment en faveur de la thèse de Charles Bally qui soutient que, dans la langue française, "l'expression verbale recule devant l'emprise croissante du substantif ${ }^{14} \gg$. M. Simpson offre à l'appui de cette déclaration un choix d'exemples dont voici un échantillon :

Je n'aurais pas confiance.

I woudn't trust it 15 .

Dans la conclusion de sa thèse, $M$. Simpson souligne une fois de plus le caractère bilingue de Beckett qui ne serait donc pas, comme l'affirme le Current Biography Yearbook, un auteur français ${ }^{16}$. In s'agit au contraire d'un authentique bilingue ayant deux cordes à son arc littéraire et dont l'œuvre est de nature à intéresser non seulement le traducteur, mais aussi le spécialiste en littérature comparée et le chercheur qui se penche sur le bilinguisme comme phénomène psychologique et linguistique. Le caractère de META, revue spécialisée, nous a amené d'ailleurs à passer sous silence une partie substantielle de la thèse qui traite de ces deux questions.

10. En attendant Godot, p. 134 ; Waiting for Godot, p. 51.

11. En attendant Godot, p. 44 ; Waiting for Godot, p. 19.

12. Happy Days, p. 12 ; Oh! les beaux jours, p. 17.

13. Happy Days, p. 24 ; Oh! les beaux jours, p. 33.

14. Linguistique générale et linguistique française, Berne, Francke, 1965, p. 346.

15. En attendant Godot, p. 26 ; Waiting for Godot, p. 12 .

16. New York, H. W. Wilson, 1970 ; voir G. E. Simpson, op. cit., p. 245. 
Comme traducteur, Beckett se situe aux antipodes de la position adoptée par Vladimir Nabokow, cas semblable à celui de Beckett et dont l'œuvre fournirait la matière d'une étude analogue à la présente thèse mais consacrée à la stylistique comparée du russe et de l'anglais. Nabokow, en traduisant l'Eugène Oniéguine de Pouchkine, opta résolument pour la traduction littérale : «Dans ma traduction, écrit-il, j'ai sacrifié à la fidélité totale et à la conservation intégrale du sens tous les éléments de la forme, sauf le rythme iambique ${ }^{17}$. » Beckett, en revanche, rejette le mot à mot pour adopter la méthode «proposée» dans la Stylistique comparée du français et de l'anglais, soit l'unité de pensée comme cadre de la traduction. Il pratique par conséquent la traduction littérale aux endroits où cela est faisable, tout en recourant aux procédés de traduction oblique quand bon lui semble ${ }^{18}$.

Quel jugement peut-on porter sur la thèse de M. Simpson ? Rappelons encore une fois qu'il ne s'agit pas d'une thèse de traduction - nos universités n'offrent pas encore de doctorat en cette matière - mais d'une thèse en études françaises qui intéresse non seulement la traduction mais aussi la littérature comparée et la linguistique. Il s'agit par conséquent d'une thèse importante, consacrée à une catégorie d'auteurs dont il n'existe que quelques rares exemples et rédigée par un candidat qui possède la compétence indispensable à l'étude d'une telle question. Car M. Simpson, détenteur d'un baccalauréat spécialisé de l'Université d'Ibadan au Nigeria et d'une maîtrise ès arts de l'Université Laval de Québec, possède également une connaissance de l'anglais et du français qui le classe parmi le nombre restreint de spécialistes capables de mener à bien un tel projet.

Par son importance, la thèse pose deux questions qui mériteraient qu'on s'y attarde. La première concerne l'opportunité de consacrer à d'autres auteurs bilingues - à Nabokow lui-même, ou à Julien Green, par exemple - des études d'une envergure semblable à celle de la thèse de M. Simpson. Il y aurait sûrement là de quoi approfondir nos connaissances non seulement en matière de traduction mais aussi sur une foule d'autres questions : littérature comparée, stylistique comparée, linguistique générale, bilinguisme, etc. À notre époque multidisciplinaire, de telles études, situées au carrefour de plusieurs disciplines, sont bien reçues et le traducteur professionnel ne serait certainement pas le dernier à profiter de tels contacts avec les spécialistes d'autres secteurs.

L'autre question concerne l'opportunité d'instituer un doctorat en traduction. Au-delà de la formation de base assurée par nos baccalauréats et nos licences actuels, la maîtrise offre une spécialisation qui est bien à sa place, compte tenu du niveau de recherches qu'elle représente, mais le travail de $M$. Simpson constitue un argument persuasif en faveur d'un cycle d'études en traduction au-delà du niveau de la maîtrise, cycle qui permettrait de mener à terme un projet de l'envergure et de la profondeur de celui que nous venons de décrire. L'acheminement des études en traduction depuis vingt-cinq ans démontre clairement les possibilités de recherche dans ce domaine. La thèse de M. Simpson indique la direction que pourrait prendre cette recherche et les dimensions qu'elle pourrait assumer.

BlaKe T. HaNna

17. "In my translation, I have sacrificed to total accuracy and completeness of meaning every element of form save the iambic rhythm. " (R. Brower (éd.), On Translation, Cambridge (Mass.), Harvard University Press, 1959, p. 97).

18. Simpson, op. cit., p. 250. 